\title{
Scoliosis Short-Term Rehabilitation (SSTR) - A Pilot Investigation
}

\author{
H Weiss, S Seibel
}

\section{Citation}

H Weiss, S Seibel. Scoliosis Short-Term Rehabilitation (SSTR) - A Pilot Investigation. The Internet Journal of Rehabilitation. 2009 Volume 1 Number 1.

DOI: $\underline{10.5580 / \mathrm{e} 71}$

\begin{abstract}
There is evidence that physiotherapy is an effective treatment module used in the management of patients with scoliosis. There is a RCT from China as well as a prospective controlled study from Germany supporting physiotherapy. The latter study was on scoliosis in-patient rehabilitation with patient samples treated intensively for 6 weeks. Today in-patient rehabilitation programs have changed and the length of the program has been reduced. Therefore, there is doubt that the results of the study are reproducible today. This is why new concepts of scoliosis rehabilitation have been developed on the basis of the latest methodological studies in this field. Material and Methods: In June 2010 the new 5-day scoliosis short-term rehabilitation was introduced based on experiential learning and the methods of treatment described in literature. 10 patients from New Zealand, US, Canada, Belarus, Rumania and Russia took part. Nine had a scoliosis, one was treated for kyphosis. The concept consisted of two guided $90 \mathrm{~min}$. sessions and one experiential learning session of another $90 \mathrm{~min}$ on a daily basis. Finally, 9 patients with the diagnosis of AIS (Adolescent Idiopathic Scoliosis), two males and seven females with an average Cobb angle of $46^{\circ}$ ( $29-$ $\left.64^{\circ}\right)$ and with an average age of $14(11-18)$ years were included. ATR (Angle of Trunk Rotation = Scoliometer) measurements were taken before and after the treatment. Additionally, the ability to correct themselves was measured after four days of treatment. A questionnaire to measure patient satisfaction was also used.Results: The ATR was reduced significantly from $10.3^{\circ}$ to $8.2^{\circ}(p<0,001)$ after treatment in the nine patients with scoliosis. The ability to correct themselves as measured with the help of the Scoliometer (ATR 8.2 ${ }^{\circ}$ / ATR auto-corrected (without additional help by the therapist) 5.7 ) was 1,45 and the difference between ATR 8.2 / ATR auto-corrected 5.7 was significant as well $(p=0,0035)$. Patient satisfaction was over average and the patient examination at the end clearly showed that all goals set were achieved for all patients.Conclusions: This new concept of rehabilitation is less time consuming and seems as effective as in-patient rehabilitation of 3-4 weeks. Therefore, in-patient rehabilitation lasting several weeks today seems necessary no more for children and adolescents with scoliosis. Studies with increasing patient samples are needed to substantiate these conclusions. Only the group of patients with scolisois is needed to be included. More explanation is needed.
\end{abstract}

\section{BACKGROUND}

There is evidence that physiotherapy is an effective treatment module used in the management of patients with scoliosis. In their systematic review Negrini and Coworkers (2008) reported on a RCT from China. There is also a prospective controlled study from Germany supporting physiotherapy in the in-patient mode (Weiss, Weiss and Petermann 2003). The latter study was on scoliosis in-patient rehabilitation with patient samples from 1989 to 1991 treated intensively for 6 weeks.

Today, the in-patient rehabilitation program has changed widely and the length of the program has also been shortened. Therefore, there is doubt that the results of the prospective study (Weiss, Weiss and Petermann 2003) are reproducible today.

The latest in-patient programs have not been investigated so far, no evidence has been achieved for their use (Yilmaz and Kozikoglu 2010) and this is why new concepts of scoliosis rehabilitation have been developed on the basis of the latest methodological studies in this field.

As early as 2006, it has been shown that the outcome of scoliosis in-patient rehabilitation can be improved by adding on the physio-logic $®$ program (Weiss and Klein 2006). Moreover, it has also been shown, that rehabilitation times can be reduced drastically (Weiss, Hollaender and Klein 2006) by adding on treatment methods based on the 
activities of daily living (ADL, see also figure 1 and 2.).

\section{Figure 1}

Fig. 1
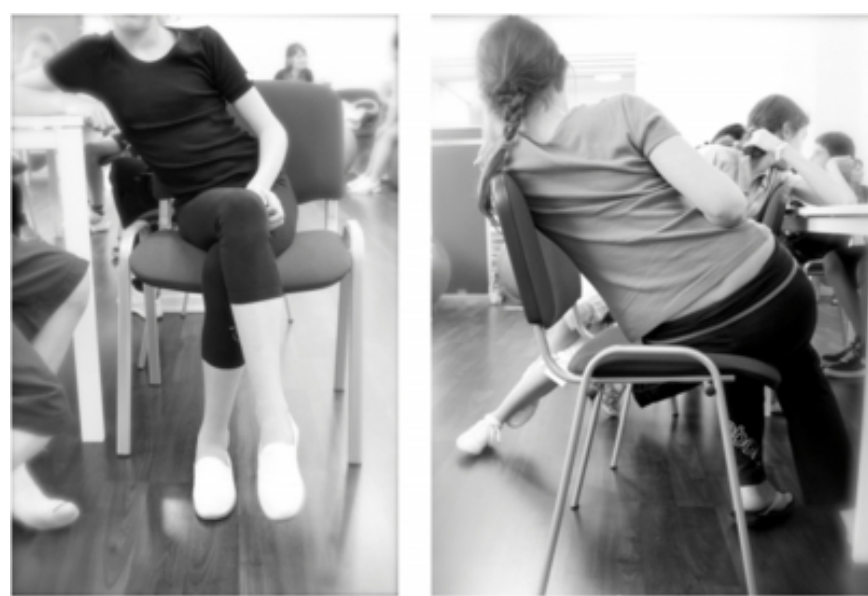

Figure 1. Correction of the activities of daily living in sitting position as performed within the SSTR Program. On the left a patient with double major scoliosis left thoracic is visible leaning to the right, opening the thoracic curvature and keeping the lumbar curvature corrected with this special position of the legs. On the right a patient with single right thoracic curvature is visible leaning to the left and by this opening the thoracic concavity. Details of this ADL correction are subject of the instructional courses given regularly. (With kind permission Pflaum, Munich)

The latest „Best Practice” program with a special methodology and a certain experiential learning approach is expected to be applied effectively within $3-5$ days. The methodology was described in the 3rd edition of the „Best Practice" textbook (Weiss 2010).

First time in history the rehabilitation of the scoliotic walk is performed naturally as well as on a special treadmill with surface analysis (Diers Formetric 4Dß). Some impressions of this new program have been submitted to YouTube and can be seen here:

http://www.youtube.com/watch?v=eHsCsL7IEaU

This video documents the SSTR course of the patient sample described within this paper.

For national patients we apply the program in 3 days, international courses of rehabilitation run 5 days in order to allow secure acquisition of skills once patients return home and the construction and application of braces during the patients stay. For national patients it is easier to be present again should any problems arise.

\section{Figure 2}

Fig. 2

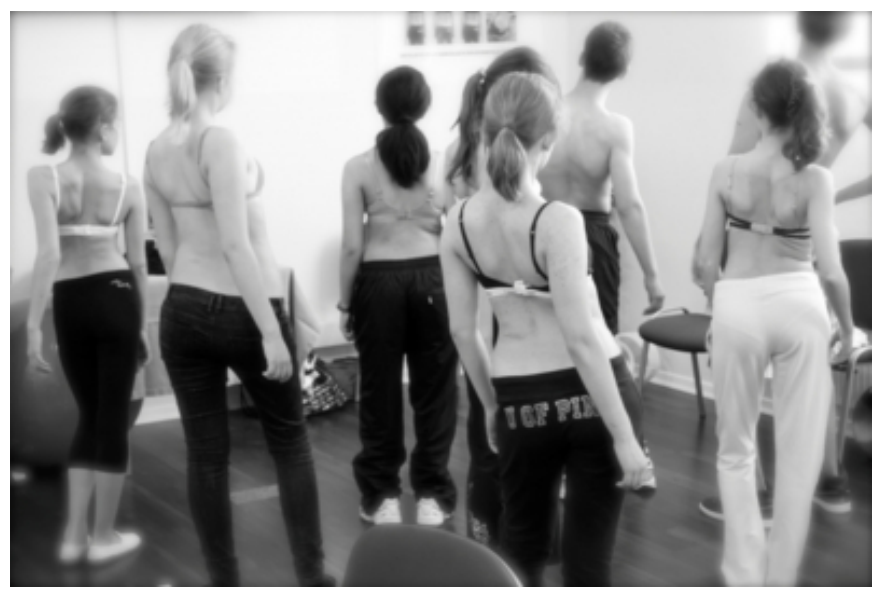

Figure 2. 3D-made-easy exercises as performed in the group. Modified from an original Photo by M. Jennings, Sydney. (With kind permission Pflaum, Munich)

\section{MATERIAL AND METHODS}

In June 2010 the new 5-day scoliosis short-term rehabilitation (SSTR) was introduced based on experiential learning and the methods of treatment described in literature (Weiss 2010). The methods applied in a special schedule with many repetitions were:

- physio-logic ${ }^{\circledR}$ program

- correction of the activities of daily living

- 3D-made-easy (Figure 2.) and

- Schroth (modified program Schroth2010 see also Figure 3.)

\section{THE PHYSIO-LOGIC EXERCISE PROGRAM}

Within the physio-logic ${ }^{\circledR}$ exercise program:

Symmetric mobilizing exercises to improve lordosing mobility of the lumbar spine and kyphosing mobility of the thoracic spine.

Asymmetric exercises to improve postural corrections also in frontal and coronal plane and

The physio-logic ${ }^{\circledR}$ ADL posture.

The symmetric mobilising exercises are performed repeatedly. These exercises can only be performed with the help of postural reflexes. Firstly lumbar lordosis is adopted actively and the pelvis is tilted forward whilst the upper 
trunk is reclined backwards to improve thoracic kyphosis by a reciprocal reflex.

It is not the aim of the exercises to increase lumbar lordosis at the L5/S1 level as in-creased stress in this region can cause lower back pain. Therefore the aim at a lordosis at the L2 level. We can ensure perfection of the exercise by ventralising the lower ribs.

For the asymmetric postural correction in 3-D we can use exercises from the Schroth program modified to the principles of the physio-logic ${ }^{\circledR}$ exercise program.

Activities of daily living (ADL) are very important to change the scoliotic stereotyped posture and for this reason the physio-logic ${ }^{\circledR}$ ADL posture is trained in standing and walking. Therefore the patients are taught to perform the "Catwalk" which includes the basic principles of the physiologic ${ }^{\circledR}$ program addressing the sagittal plane and the ADL (Activities of daily living) posture.

The physio-logic ${ }^{\circledR}$ program can be used for the treatment of small curves $\left(15-20^{\circ}\right)$ and for the treatment of back pain as well, when the sagittal profile shows a mal-alignment (Weiss and Klein 2006).

\section{PATTERN SPECIFIC ADL}

The pattern specific ADL in some way are similar to the side-shift exercises (Weiss, Hollaender and Klein 2006). These have been applied in two different exercises, one for thoracic curves and one for lumbar curve patterns.

In the "Best Practice" program three key patterns, which we address individually:

- Thoracic curve pattern

- Double major curve pattern

- Lumbar / thoracolumbar curve pattern

The pattern specific ADL should accompany every other approach as described here.

\section{D-EXERCISES MADE EASY}

The "3D-Exercises made easy" program is derived from the activities of daily living (ADL). These exercises can be performed in the sitting and standing position. A thoracic, a lumbar and a double major exercise can be performed addressing the different curve pattern in 3D. The thoracolumbar curve patterns can be addressed by applying the thoracic exercise (High thoracolumbar curve with apex $\mathrm{TH}$ 12) or the lumbar exercise (Low thoracolumbar curve with apex L1).

The "3D-Exercises made easy" have been demonstrated to be easy to teach (Weiss, Hollaender and Klein 2006) and can be used for the treatment of small curves $\left(15-30^{\circ}\right)$ together with the physio-logic ${ }^{\circledR}$ program (Weiss and Klein 2006).

\section{THE SCHROTH PROGRAM}

The cognitive behavioral exercise program according to Schroth is described at length in several publications (Weiss 2010) and the idea of in-patient rehabilitation has been subject of many scientific investigations (Weiss 2010). The advantage of this program is the specificity of postural corrections designed for different curve patterns and the introduction of effective mechanisms to increase 3-D postural correction in scoliosis.

The basic aim of physiotherapy is to enable the patient to attain postural corrections themselves, with the exclusive use of trunk muscles, although dynamic passive forces (FED system- manual help by the therapist) and static forces (passive correction using bean bags) are applied. In order to train the patient, the various types of assistance offered by the therapist are essential. Firstly, the therapist instructs the patient. The patient is also taught how to make slight corrections or adjustments to his or her posture in different parts of the body by means of exteroceptive stimulation or by provoking a reaction of balance. Equally important are the proprioceptive stimulations, both through manual passive corrections on the deformed trunk and through changes in articular position, passive traction-compression movements or manual relaxation, activation or elongation tests of elastic structures.

Within the Schroth exercise program, pattern-specific correction mechanisms are taught depending on the clinical findings of the patient. Although the number of possible curve patterns seems quite high, in the Schroth system four basic curve patterns are considered, which in practice seems enough to address most of the typical findings a scoliosis can present with:

- Functional 3 curve pattern with neutral pelvis

- Functional 3 curve pattern with decompensation

- Functional 4 curve pattern, and as a special form of the 4 curve pattern the Thoracolumbar curve pattern 
10 patients from New Zealand, US, Canada, Belarus,

Rumania and Russia took part. Nine had a scoliosis, one was treated for kyphosis. The patient with kyphosis was excluded from the study.

\section{Figure 3}

Fig. 3

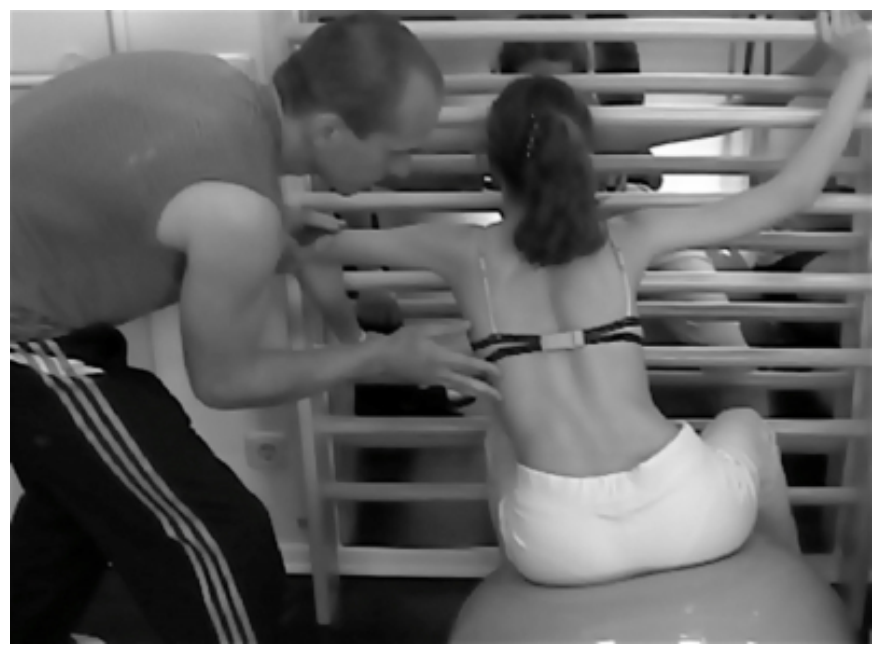

Figure 3. New Schroth exercise using the synergy effects of the arms differently in order to correct rib hump and flat back at the same time. (With kind permission Pflaum, Munich)

Finally, 9 Patients, diagnosed with AIS (Adolescent Idiopathic Scoliosis), two males and seven females with an average Cobb angle of $46^{\circ}\left(29-64^{\circ}\right)$ and with an average age of $14(11-18)$ years were included.

The concept consisted of two guided 90 min. sessions and in between one experiential learning session of another $90 \mathrm{~min}$. daily.

\section{ATR $($ Angle of Trunk Rotation $=$ Scoliometer $)$} measurements were taken before and after the treatment. Additionally, the ability to correct themselves as measured with the help of the Scoliometer was recorded after four days of treatment (ATR / ATR auto-corrected corrected without additional help by the therapist).

An examination sheet was provided to estimate patient's knowledge about their individual curve pattern and about the way to correct this specifically with the exercises (appendix 1).

We have established a Spinal Deformity Rehabilitation Outcome Score (SDROS) for this special treatment in order to allow a rating of skill acquisition and performance $(0-$ 10):
- physio-logic ${ }^{\circledR}$ program performed correctly (2 Points)

- correction of the activities of daily living performed correctly (2 Points)

- 3D-made-easy and Schroth performed correctly (2 Points)

- Improvement of the ATR (Scoliometer) (2 Points)

- Acquisition of the necessary knowledge (examination) (2 Points)

- A questionnaire to measure patient satisfaction was used additionally (see appendix 2).

\section{RESULTS \\ MEASUREMENTS}

The ATR was reduced significantly from $10.3^{\circ}$ to $8.2^{\circ}$ (p < $0,001)$ after treatment in the nine patients with scoliosis. The ability to correct themselves as measured with the help of the Scoliometer (ATR 8.2 / ATR auto-corrected 5,7 (corrected without additional help by the therapist)) was 1,45 and the difference between ATR 8.2० / ATR auto-corrected 5.7 was also significant $(\mathrm{p}=0,0035)$. Patient satisfaction subjectively was good and the patient examination at the end clearly showed that all goals were achieved for all patients.

\section{SDROS}

All nine patients reached the maximum of 10 points. The examination sheet can be seen as appendix 1 .

Questionnaire (see appendix 2)

- -The answers in the questionnaire for question 1 (During the intensive short-term rehabilitation I have learnt a lot, which can be applied in everyday life) were:

- applies 8 , does not apply 1 .

- -The answers in the questionnaire for question 2 (I feel absolutely safe and secure to be able to perform the appropriate exercises at home) were:

- applies 8 , does not apply 1 .

- -The answers in the questionnaire for question 3 (I will concentrate on the correction of my daily activities in the future) were: 
- applies 8, applies in part 1.

- -The answers in the questionnaire for question 4 (I will perform the exercises every day for at least 30 min. during the first two weeks until I feel safe with my activities of daily living) were:

- applies 7, applies in part 1,does not apply 1 .

- -The answers in the questionnaire for question 5 (Within this short-term rehabilitation program I have learnt much more about postural control than with any other program previously) were:

- applies 8, applies in part 1 .

- -The answers in the questionnaire for question 6 (I would suggest other patients with scoliosis to undergo this kind of short-term rehabilitation) were:

- applies 6, applies in part 3 .

\section{DISCUSSION}

All ATRs improved and this is why even in this small sample of patients the changes reached a significant level. Additionally, for the first time auto-correction (without additional help by the therapist) was tested with two of the 9 patients not able to autocorrect further.

The results of Scoliosis in-patient rehabilitation for postural changes were significant in huge samples, but not relevant at average. In a survey on $>1000$ patients with scoliosis measured with the help of surface topography (Diers, Formetric ( ) lateral deviation improved at average $1 \mathrm{~mm}$ after a four week course of in-patient rehabilitation (Weiss, Verres and El Obeidi 1999) the average improvement still within the range of technical error (Weiss, Lohschmidt and El Obeidi 1997). Significant improvements of ATR as achieved after in-patient rehabilitation have not yet been reported.

Considering the fact, that the retrospective results of inpatient rehabilitation substantially were not better than for out-patient in terms of physiotherapy, the question arises, as to whether there is need for in-patient rehabilitation for children and adolescents with scoliosis at all. Yilmaz and Kozikoglu (2010) recently doubted the effect of in-patient rehabilitation to be over the effects of outpatient programs.
The physical improvements achieved within this study did not correlate well in one patient with the subjective rating. The 12 year old girl with a double major curve and a still undiagnosed neurological problem (accidental fainting) has had chronic low back pain, which her mother did not report before the start of treatment. The aim for this girl subjectively was to get rid of the pain problems.

However, pain increased during the treatment sessions, were then relieved by manual therapy, but returned when performing the specific exercises. Nevertheless in the brace adjusted during SSTR she is pain free and therefore wears it full time.

It is basically true that there is a prospective controlled study on in-patient rehabilitation, but today there is also a randomized controlled study which speaks for out-patient physiotherapy and this has to be regarded as being of higher evidence. Furthermore, the study on in-patient rehabilitation was on patient samples treated intensively for 6 weeks. Meanwhile, programs have changed and the rehabilitation times have been reduced. This is why no evidence can be claimed for today's in-patient rehabilitation.

The promising results of this pilot investigation are encouraging and therefore we will gather more information about patient outcomes and ratings in future courses. Inpatient programs today seem an unnecessary endeavor for children and adolescents with idiopathic scoliosis not complicated by other health related issues (Yilmaz and Kozikoglu 2010).

The ScoliOlogiC Best Practice program leads to a safe acquisition of skills necessary for the patient to enable proper performance of the exercises at home. Postural feeling is improved and auto-correction maneuvers can easily be reproduced. The physio-logic $\AA$ program leads to a relaxed and, at the same time upright posture without the need for trunk muscle tension. This is what the patients were able to experience within a short period of time. The physiologic $₫$ posture has not yet been analyzed biomechanically. This is why we plan a study with Formetric $®$ surface topography (Weiss HR , Lohschmidt and El Obeidi 1997) in different postural positions in due course.

The rehabilitation of the scoliotic patterns of walking was performed within this program for the first time. We do believe that the postural correction during ambulation is a useful tool and the patients were able to acquire this dynamic correction easily. 


\section{Figure 4}

Fig 4.
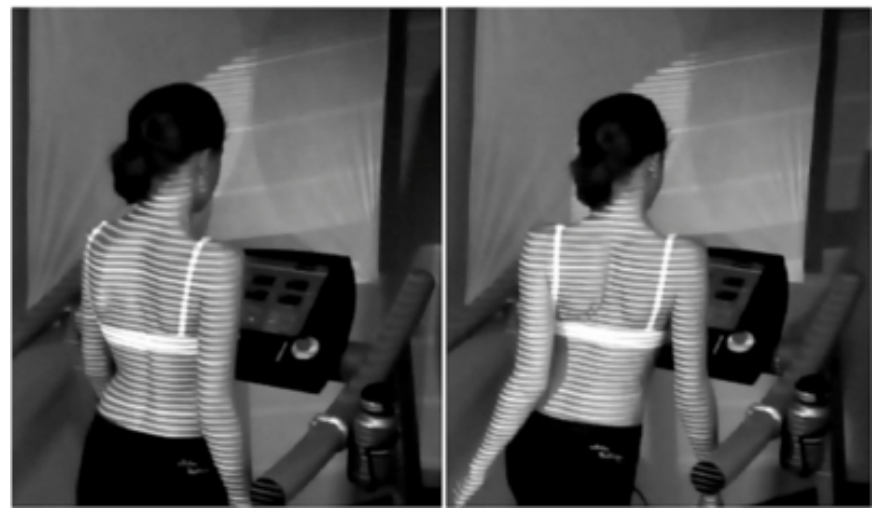

Figure 4. Correction of scoliotic walking pattern on a tread mill using dynamic 3D reconstruction with the help of the Formetric surface scanner. (With kind permission Pflaum, Munich)

The future will tell as to whether the international community of experts will be able to accept the measure of the ability of patients to correct themselves as measured with the help of the Scoliometer as introduced within this study. Nevertheless, the ATR measurements before and after the program as well as the measurements before and after the correction maneuver at the end of treatment were better than the technical error $\left(1^{\circ}\right)$, (Weiss and Lehnert-Schroth 1994).

\section{CONCLUSION}

This new concept of rehabilitation is less time consuming and seems as effective as in-patient rehabilitation of 3-4 weeks. Therefore, in-patient rehabilitation today probably is outdated for children and adolescents with scoliosis.

Nevertheless, studies with increasing patient samples are needed to substantiate these conclusions.

\section{COMPETING INTERESTS}

The first author is applying for a patent for a brace not relating to the content of this paper and is advisor of KoobScolitech, Abtweiler, Germany.

SS declares to have no competing interest.

\section{AUTHORS' CONTRIBUTIONS}

HRW: Analysis and interpretation of data, preparation of the manuscript, acquisition of pictures and additional materials, corresponding author

SS: Patient investigation, scans, $x$-rays, assistance in study management.

\section{ACKNOWLEDGEMENTS}

The authors are thankful to Orthomed, Orthopedic Technical Services, Gensingen, Germany, for their kind support.

Many thanks as well to Matthew Jennings, Syndey, Australia for providing a number of photographs as a participant of the course.

The authors wish to thank Lesley Schneider for copyediting this paper.

Written informed consent was obtained by all persons visible on the pictures submitted.

\section{APPENDIX 1.}

\section{Figure 5}

\section{Examination sheet}

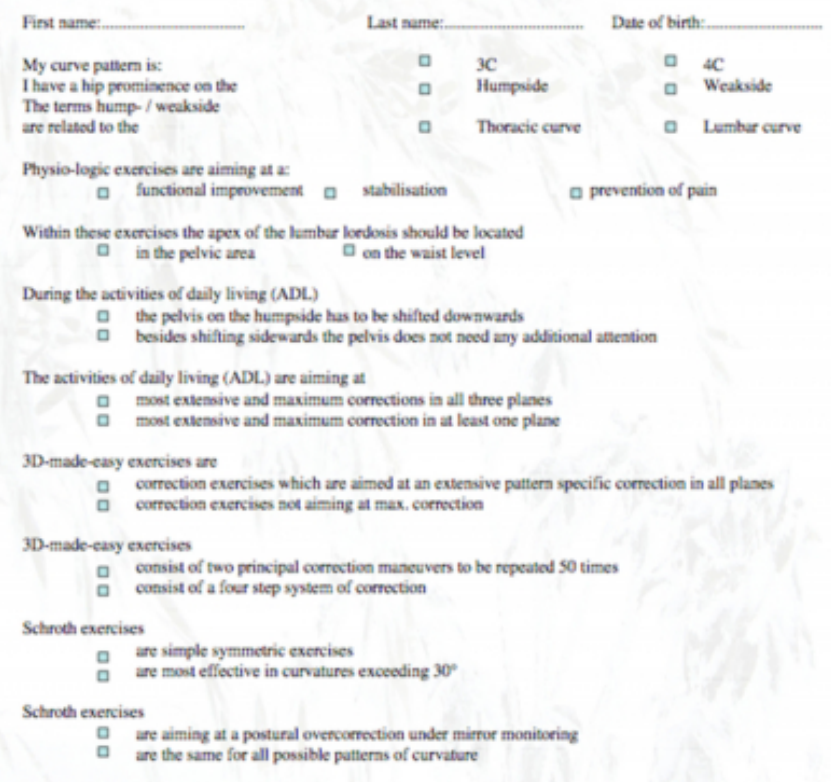

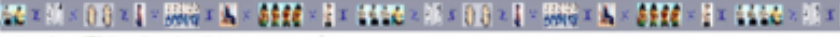

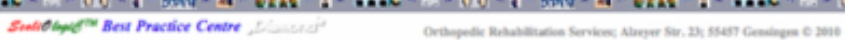

APPENDIX 2. 


\section{Figure 6}

\section{Questionnaire (please circle the appropriate item)}

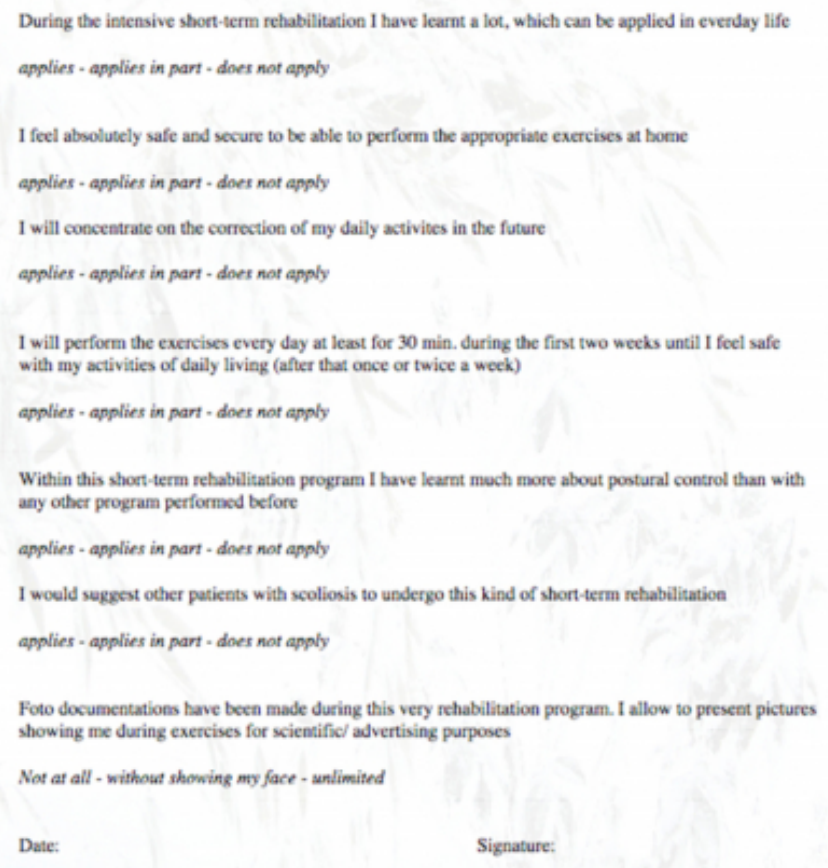

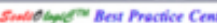

\section{References}

1. Negrini S, Fusco C, Minozzi S, Atanasio S, Zaina F, Romano M: Exercises reduce the progression rate of adolescent idiopathic scoliosis: results of a comprehensive systematic review of the literature. Disabil Rehabil.; 2008; 30(10): 772-85

2. Weiss HR: Best Practice in conservative scoliosis care. 3rd. edition Pflaum, Munich; 2010

3. Weiss HR , Klein R: Improving excellence in scoliosis rehabilitation: a controlled study of matched pairs. Pediatr Rehabil; 2006; 9: 190-200 Jul/Sep

4. Weiss HR, Hollaender M, Klein R: ADL based scoliosis rehabilitation--the key to an improvement of timeefficiency? Stud Health Technol Inform; 2006; 123: 594-598 5. Weiss HR, Weiss G, Petermann F: Incidence of curvature progression in idiopathic scoliosis patients treated with scoliosis in-patient rehabilitation (SIR): an age- and sexmatched controlled study. Pediatr Rehabil; 2003; 6: 23-30 Jan/Mar

6. Weiss HR, Verres C, El Obeidi N: Ermittlung der Ergebnisqualität der Rehabilitation von Patienten mit Wirbelsäulendeformitäten durch objektive Analyse der Rückenform. Phys Rehab Kur Med; 1999; 9: 41-47

7. Weiss HR, Lohschmidt K, El Obeidi N: The Automated Surface Measurement of the Trunk. Technical Error. In: Research into Spinal Deformities I, Edited by:J.A. Sevastik and K.M. Diab. 305-308 IOS Press, Amsterdam 1997 8. Weiss HR , Lehnert-Schroth Ch: Chest Movements in Scoliosis Patients In: Proceedings of the Fifth Biannual Conference of the ESDS in Birmingham, 31st May-3rd June 106-107 Biannual Conference of the ESDS Birmingham 1994

9. Yilmaz H, Kozikoglu L: Inpatient rehabilitation - A systematic Pub Med review. The Internet Journal of Rehabilitation. 2010 Volume 1 Number 1 


\section{Author Information}

Hans-Rudolf Weiss, MD

Sarah Seibel 